\title{
Modelling of plasma-edge and plasma-wall interaction physics at JET with the metallic first-wall
}

\author{
$\underline{\text { S. Wiesen }}^{1,2^{*}}$, M. Groth ${ }^{3}$, S.Brezinsek $^{1}$, M. Wischmeier ${ }^{4}$ and JET contributors ${ }^{\dagger}$ \\ EUROfusion Consortium, JET, Culham Science Centre, OX14 3DB, Abingdon, UK \\ 1) Forschungszentrum Jülich GmbH, Institut für Energie- und Klimaforschung - \\ Plasmaphysik, 52425 Jülich, Germany \\ 2) Culham Centre for Fusion Energy, Culham Science Centre, Abingdon, OX14 3DB, \\ $U K$ \\ 3) Aalto University, Otakaari 1, 02150 Espoo, Finland \\ 4) Max-Planck-Institut fuer Plasmaphysik, Boltzmannstr. 2, D-85748 Garching, \\ Germany
}

\begin{abstract}
An overview is given on the recent progress on edge modelling activities for the JET ITERlike wall using the computational tools like the SOLPS or EDGE2D-EIRENE code. The validation process of these codes on JET with its metallic plasma-facing components is an important step towards predictive studies for ITER and DEMO in relevant divertor operational conditions, i.e., for detached, radiating divertors. With increased quantitative credibility in such codes more reliable input to plasma-wall and plasma-material codes can be warranted, which in turn results in more realistic and physically sound estimates of the lifetime expectations and performance of a Be first-wall and a W-divertor, the same materials configuration foreseen for ITER. A brief review is given on the recent achievements in the plasma-wall interaction and material migration studies. Finally, a short summary is given on the availability and development of integrated codes to assess the performance of an JET-ILW baseline scenario also in view of the preparation for a JET DT-campaign.

PACS: 52.30.Ex, 52.40.Hf, 52.55.Fa, 52.55.Rk, 52.25.-b

\footnotetext{
*Email corresponding author: s.wiesen@fz-juelich.de

${ }^{\dagger}$ See the Appendix of F. Romanelli et al., Proceedings of the 25th IAEA Fusion Energy Conference 2014, Saint Petersburg, Russia
} 


\section{Introduction}

JET with its metallic wall consisting of a beryllium first-wall and tungsten armour in the divertor (ITER-like wall, ILW [1,2]) has demonstrated to perform very successfully for plasma-wall interaction studies and plasma operation with the identical plasma-facing material selection foreseen in ITER [3]. It has been proven that with the ILW in JET the goal to minimise long-term fuel retention can be achieved [4,5] and that the plasma-facing components (PFC) also do allow for a fast isotope exchange [6]. The JET ITER-like wall material configuration setup provides a unique set of experimental data which is also exploited for the validation of numerical models extensively used to predict the life-time and performance of the ITER plasma-facing components.

Since the installation of the ILW in JET in 2010 and driven by a dedicated validation process there has been significant progress in the understanding of the plasma-edge physics that includes scrape-off-layer (SOL) and divertor plasma transport, as well as the inseparable link to the plasma-wall interaction physics. To some extent this is nowadays more straightforward because in metallic devices, like the JET-ILW or ASDEX-Upgrade (AUG, with full-W wall) the lack of intrinsic carbon radiation cannot conceal relevant physics processes anymore.

In this paper an overview is given on the highlights on the validation progress of edge modelling tools, i.e. the advance of modelling capabilities for the plasma edge using SOLPS, EDGE2D-EIRENE and other similar codes that includes the following topics: divertor detachment, seeded discharges in low and high confinement modes (L-mode and H-mode), SOL/edge plasma transport and (sub-) divertor neutral transport. Any such improvement on the SOL and edge plasma modelling capabilities allows automatically further progress also on other models linking plasma-wall interaction (PWI) or plasma-material interaction (PMI) processes with the actual plasma, e.g. models for material transport of particle eroded from the Be-limiter first wall towards the W-divertor PFCs. Some highlights are presented on the exploitation of wall erosion codes like ERO, DIVIMP and global material transport codes, i.e. WALLDYN. In view of the upcoming DT-campaign at JET, currently foreseen in 2017/18, an outlook is given about the readiness of the integrated modelling codes available, necessary for the prediction and analysis of JET DT scenarios. Recent progress in the development and extension of the used codes are highlighted. To conclude some remarks on remaining lack of understanding for some key physics aspects are given. They include, for example issues on the power and particle exhaust problem which are important for a successful extrapolation 
from existing devices like JET towards ITER or DEMO.

\section{Particle and power exhaust}

Recent studies utilising infra-red measurements to determine the target heat fluxes in JET or ASDEX-Upgrade (AUG) have shown that a unique parametrisation of the target heat flux profile can be derived [7]. Essentially, the heat flux profile consists of a convolution of an exponential decay and a Gaussian function simultaneously describing the decay of the heat flux into the common SOL and into the private flux region. An important machine parameter to measure the effectiveness of the power dissipation process in a divertor can be expressed by its maximum power density $\mathrm{q}_{\max } \sim \mathrm{P}_{\mathrm{div}} /\left(\mathrm{R} \lambda_{\text {int }}\right)$, where $\mathrm{P}_{\mathrm{div}}$ is the power arriving in the divertor, $\mathrm{R}$ is the major radius and $\lambda_{\text {int }}$ is the (measurable) integrated heat flux parameter along the target coordinate $s$, i.e. $\left.\lambda_{\text {int }}=\int\left(q(s)-q_{0}\right) d s\right) / q_{\max }$. It was found that this parameter can be simply reduced to a linear combination of $\lambda_{\mathrm{q}}$, the upstream heat flux decay parameter, and a dissipation parameter S, i.e. $\lambda_{\text {int }}=\lambda_{\mathrm{q}}+1.64 \mathrm{~S}$ [8]. This simple scaling formula for $\lambda_{\text {int }}$ was found to be valid for many tokamaks for low- to medium-density plasmas, with an inverse dependence of the upstream heat decay parameter on the poloidal magnetic field $\lambda_{\mathrm{q}} \sim 1 / \mathrm{B}_{\mathrm{p}}$, i.e. no dependency on machine size [9]. Thus, for ITER one can derive an upstream heat decay parameter $\lambda_{\mathrm{q}} \sim 1 \mathrm{~mm}$. Thus the critical constraint for the plasma-facing component (PFC) heat loads of about 5-10 MW/ $\mathrm{m}^{2}$ [10] can only be met by making power dissipation (the $\mathrm{S}$ parameter) within the divertor large. Recent complementary SOLPS modelling on AUG and JET [11] suggests a dissipation parameter scaling $\mathrm{S} \sim 1 / \mathrm{T}_{\mathrm{e}}{ }^{\text {plate }}$. This qualitatively restates the fact that ITER must operate at low divertor temperature to ensure long-term survival of the target plate PFCs, i.e. by operating a partially detached divertor and potentially allowing for enhanced radiation loss in the SOL/divertor region. Since in metallic devices carbon as main radiator at SOL characteristic temperatures is missing, extra radiation can only achieved by seeding impurities, i.e. N2, Ne, Ar etc.

It is important to acknowledge the process of the power dissipation mechanism in divertor configurations. Essentially it can be described as a step-ladder process of removing (or redirecting) plasma energy and particles as it is transported along the magnetic field lines in the SOL towards the divertor [12]. Power entering the SOL by anomalous (ballooning-like) cross-field transport from the confined region is conducted mainly by T-gradients along the field lines until it reaches an impurity radiation zone, which reduces temperature and thus 
heat-flux. With decreasing $\mathrm{T}_{\mathrm{e}}$ and after passing the ionization front at $\mathrm{T}_{\mathrm{e}} \sim 5 \mathrm{eV}$ pressure is removed from the plasma by friction processes with the compressed divertor neutrals and further T-reduction is a consequence, as well as plasma particle loss by charge-exchange (CX) processes. If sufficient power could be radiated or dissipated before reaching the target plate $\mathrm{T}_{\mathrm{e}}$ is reduced below $5 \mathrm{eV}$, and at $\mathrm{T}_{\mathrm{e}}<2 \mathrm{eV}$ strong recombination processes will cause further loss of plasma particles. The transition into detachment is characterised by a roll-over of the plate saturation current (i.e. the particle-flux), which will effectively reduce the target heat load by fewer plasma particles recombining at the plate (i.e., a smaller number of particles will deposit their recombination energy). Other particle loss terms like enhanced radial transport or energy loss by charge-exchange or other atomic processes, like molecular assisted reactions are also capable to increase the degree of detachment $[13,14,15]$. It is widely accepted that for the highly non-linear process of power dissipation by divertor detachment no general scaling is available (c.f. [16] and references therein). Thus for extrapolation towards ITER or DEMO it is of utter importance to validate the existing edge and divertor plasma codes like SOLPS [17] or EDGE2D-EIRENE [18,19,20] against existing devices, and if not successful, to improve the applied numerical models. If unsuccessful, abandoning of existing codes may be necessary if the numerical model is unable to reflect the physics properly.

Since the installation of the ILW in JET strong contributions were made to allow an improved confidence in the applicability of edge codes, qualitatively and quantitatively. A long outstanding issue in the numerical assessment of the transition into detachment was a lack of understanding of the SOL flows and plasma-molecule interactions. EDGE2DEIRENE simulations of unseeded JET-ILW L-mode discharges in vertical (VT) and semihorizontal target (HT) divertor configurations have shown [21] that by neglecting cross-field drifts in both, the common-flux zone (CFZ) and the private-flux zone (PFZ), one does overestimate the high-field side (HFS) plate $\mathrm{T}_{\mathrm{e}}{ }^{\mathrm{HFS}}$ in low-recycling/attached divertor conditions and that $\mathrm{T}_{e}{ }^{\mathrm{HFS}}$ follows rather the monotonic negative slope of $\mathrm{T}_{\mathrm{e}}{ }^{\mathrm{LFS}}$ at the low-field side (LFS) as function of upstream density $\mathrm{n}_{\mathrm{e}}{ }^{\text {sep }}$. The model does reproduce the target particle flux roll-over but at a lower density compared to the experiment.

The inclusion of cross-field drifts does help to redistribute the plasms flows arriving at the target plates to be strongest in the high-recycling regime as it was shown using SOLPS5 simulations for ASDEX-Upgrade (AUG, with a full-W wall) [22] and also for JET-ILW $[23,24]$. In agreement with Langmuir probe (LP) data the model does overestimate $\mathrm{T}_{\mathrm{e}}{ }^{\mathrm{HFS}}$ by at least a factor 2 in low-recycling conditions only, also the particle fluxes clearly become asymmetric. The particle flux roll-over as function of $\mathrm{n}_{\mathrm{e}}{ }^{\mathrm{sep}}$ occurs earlier at the HFS compared 
to the LFS which qualitatively reflects the experimental observations. Also in EGDE2DEIRENE simulations for the JET-ILW but with activated cross-field drifts included $[25,26]$ a similar trend for SOL-flow asymmetry was found. Only recently a detailed discrimination of the relavant drift effects was pursued using EDGE2D-EIRENE comparing also reversed-Bt (ion grad B drift away from X-point) with normal-Bt field configurations (ion grad B drift toward X-point) [27]. The main finding is that in the PFZ the poloidal ExB drift is dominant whereas in the CFZ the radial ExB counterpart is more significant. The combined action of both ExB components does lead to the observed SOL flow asymmetry.

It is important to note that the inclusion of drift effects in edge codes can put higher demand in computational performance. It is a known fact amongst edge modellers that the time-step assumed in codes like EDGE2D-EIRENE or SOLPS must be low (i.e. $\sim 10^{-7} \mathrm{~s}$ or even lower $<10^{-8} \mathrm{~s}$ ) in order to make the simulation stable with drifts included. Some modellers artificially scale down the drifts in the model in the beginning and drive slowly in to the full drift model at a later stage of the simulation. Depending on the assumed convergence criteria a numerical solution might or might not exist and, as a general rule, it is up to the "pilot" of the code to interpret the code results correctly.

In metal devices the radiation by the main plasma species only (e.g., deuterium Lyman and Balmer line radiation or by D2 molecules) is insufficient to redistribute a significant fraction of the power density $\mathrm{q}_{\|}$carried along the field lines into the divertor. Hence external impurity seeding is needed to reduce $\mathrm{q}_{\|}$allowing for a deposition of radiated heat on remote areas. For JET-ILW H-mode discharges it was successfully shown that the maximum of $\mathrm{q}_{\|}$ arriving at the LFS plate, $\mathrm{q}_{\text {peak }}$ LFS, decreases with $\mathrm{Ne}$ or $\mathrm{N}$ injection rates [28] and the accompanying EDGE2D-EIRENE simulations [29] are in qualitative agreement with the trend of decreasing $\mathrm{q}_{\text {peak }}{ }^{\mathrm{LFS}}$ at the plate with increased radiative power fraction $\mathrm{f}_{\text {rad, }}$, the latter defined by the ratio of power radiation in the divertor including the $\mathrm{X}$-point $\left(\mathrm{P}_{\text {rad }}{ }^{\text {div }}\right)$ divided by power entering the CFZ, $\mathrm{P}^{\mathrm{sep}}$. Quantitatively, $\mathrm{f}_{\text {rad }}$ is consistently underestimated by approximately $10 \%$ only in the model when compared to the bolometry, however this discrepancy could be related to uncertainties in the measurements or due to the assumed constant level of anomalous transport in the EDGE2D-EIRENE model or both. It needs to be stressed that deuterium radiation is generally underestimated by a factor 2-3 in EDGE2DEIRENE or SOLPS simulations for unseeded discharges [30, 29]; the reason for this radiation shortfall in the code is not fully understood. Nevertheless, the model results demonstrated very clearly that $\mathrm{N}$ radiates dominantly in the divertor whereas $\mathrm{Ne}$ radiates closer to the pedestal region. Although the model does also show that inclusion of transport effects into the 
calculated radiative power function $\mathrm{R}\left(\mathrm{T}_{\mathrm{e}}\right)\left[\mathrm{W} / \mathrm{m}^{3}\right]$ reveals a broader parametric dependence on $T_{e}$, i.e. shifting the peak if $R\left(T_{e}\right)$ towards higher $T_{e}$, the overall radiation pattern in $\mathrm{N}$ - or $\mathrm{Ne}-$ seeded JET-ILW discharges is clearly reproduced. Similar quantitative results have been obtained with SOLPS5 including cross-field drifts which demonstrate that at the maximum attainable $\mathrm{f}_{\mathrm{rad}} \sim 60-70 \%$ induced by N-seeding in JET-ILW both HFS and LFS divertors are clearly detached leading to a minimum $\mathrm{q}_{\mathrm{peak}}$ at the plate [24]. It is a major achievement that both SOLPS5 and EDGE2D-EIRENE do reproduce the sequence into seeded divertor detachment at least qualitatively and to some extent also quantitatively.

Critical for the transition into detachment is a sufficient large neutral pressure $\mathrm{p}_{0}$ in the divertor to remove parallel ion momentum by neutral friction. The divertor presure $\mathrm{p}_{0}$ can be regarded as an operational parameter to control the level of detachment, for example in ITER [31]. Contrary to AUG, JET-ILW measurements of $\mathrm{p}_{0}$ in the direct vicinity of the divertor are missing and only a single Penning gauge in the sub-divertor is available. It was only until recently that a concerted attempt to model the sub-divertor pressure $\mathrm{p}_{0}{ }^{\text {sub-div }}$ by using an extended grid model for the neutrals in the EIRENE code has been pursued. In the extended model EIRENE follows Monte-Carlo particle histories down to the sub-divertor structures including the pathway up to the cryo-pump [32]. It was found that $\mathrm{p}_{0}{ }^{\text {sub-div }}$ depends on upstream $n_{e}$ sep and thus on the applied gas-flux $\Phi_{D}$. This direct correlation, for the first time, could be reproduced by a code like EDGE2D-EIRENE for JET. It was also demonstrated that as in the experiment factors of 2-5 higher $\mathrm{p}_{0}{ }^{\text {sub-div }}$ in VT configuration requires higher fuelling $\Phi_{\mathrm{D}}$ compared to a semi-horizontal (HT) configuration. Another interesting feature for the HT configuration was also found with the new EIRENE grid extension: a clear observation of a net neutral flux entering the divertor from the HFS divertor throat by particles recirculating via the sub-divertor, an effect not observed however in the model with VT configuration [32]. For lower density cases the same/similar dependence was obtained with the DIVGAS code utilising Direct Simulation Monte Carlo (DSMC, [33]), and both DIVGAS and EIRENE were positively benchmarked against each other [34]. For higher densities and detached JET-ILW divertors the DSMC model conjectures a significant effect of the neutral viscosity at Knudsen

numbers $\mathrm{Kn}<1$. However, a saturation of $\mathrm{p}_{0}{ }^{\text {sub-div }}$ with $\Phi_{\mathrm{D}}$ as observed in the experiments is not recovered in the DIVGAS simulations [34].

\section{Plasma-Wall interaction}

What makes the JET-ILW unique is the fact that it is currently the only device capable of 
addressing ITER relevant Be main-chamber wall (MCW) life-time predictions. Be material can be removed from the MCW by plasma-wall interactions (PWI), i.e. by the plasma in direct contact with the wall during the flat-top phase of the plasma but also during strong magneto-hydrodynamic events like ELMs (edge-localised modes) or plasma disruptions. Fast charge-exchange neutrals can also erode particles significantly. Eroded Be material can be transported with the plasma towards remote areas and deposited, for example, into the divertor. Run-away electrons created after disruptions can lead to localised melting and splashes and thus produce material flaking. As a consequence Be dust can accumulate in the device, which is a general safety issue for ITER [35]. Furthermore, at increased divertor heat loads the JET W-PFCs can undergo serious structural modifications, for example delaminations of $\mathrm{W}$-coated CFC tiles have been observed [36]. W-sputtering is significant at ELM induced particle and heat loads [37, 38] which needs to be minimised to keep W concentration, $\mathrm{c}_{\mathrm{W}}$, in the confined plasma sufficiently low, i.e. $\mathrm{c}_{\mathrm{W}}<10^{-5}$.

For actual predictions of the material migration mechanism the ERO Monte-Carlo code [39] or the DIVIMP trace-impurity code [40] are being employed. For dust transport studies codes like DTOKS [41] or DUSTRACK are currently being benchmarked vs JET-ILW data [42]. On a more global scale the WallDYN code [43] does follow the evolution of material transport by mapping source and deposition profiles over time across the whole device. All these codes have in common that they depend on a) reliable model databases for the material source, i.e. yields consistent with the measured influx off the PFCs in existing experiments, and b) validated plasma backgrounds for diverted plasmas with the plasma properly extrapolated up to the first wall and other remote areas. As for the edge codes, also the plasma-material and material migration codes need to undergo dedicated validation processes to allow for reliable ITER predictions.

Recently, a strong effort was made to validate and thus to improve the implemented databases in material transport codes, which are essentially databases for particle reflection coefficients and yields of Be- or W-particles sputtered by main or impurity species and depending angular and energy distribution functions. For the Be source, spectroscopic measurements of JET-ILW limiter experiments allowed for a reliable comparison with artificial diagnostics available in ERO and a lack of BeI or BeII light has been observed in the original ERO model [44], hence an underestimate of the total Be yield was observed in the code. Recent molecular dynamics (MD) simulations predicted however that a second channel to produce $\mathrm{Be}$ via chemically assisted processes producing $\mathrm{Be}_{\mathrm{x}}$ molecules does exist on top of the atomic Be yield [45, 46, 47]. Furthermore these MD-simulations have shown that the 
$\mathrm{BeD}_{\mathrm{x}}$ production decays with increasing baseplate temperature $\mathrm{T}_{\text {base }}$ of the Be-tile, which was also recovered by the analysis of molecular BeD spectra [48]. By taking these new results into account in ERO a much better comparison of the spectroscopic data within a factor of 2 was possible [48], which reproduced also negative dependence of the $\mathrm{BeD}_{\mathrm{x}}$ yield with $\mathrm{T}_{\text {base }}$ and also recovered strong Be self-sputtering at temperatures $\mathrm{T}_{\mathrm{e}}>30 \mathrm{eV}$.

A necessary ingredient when addressing the actual material transport in diverted JETILW configurations by ERO or WallDYN is a validated plasma background, which is usually prepared by running SOLPS or EDGE2D-EIRENE. However, the plasma grid in these codes does currently not extend to the main-chamber wall (contrary to the neutrals which can interact with all PFCs in the coupled EIRENE code) and hence some assumptions are necessary to extrapolate the plasma towards the first wall. With such assumptions the global impurity transport code WallDYN was benchmarked against experiments in JET-ILW [49]. In a comparison with $\mathrm{W}$ erosion and deposition calculations to post mortem analysis of $\mathrm{W}$ deposition WallDYN matched the qualitative deposition pattern well. The quantitative comparison, however, falls short by a factor 20 , which could be related to two factors: a) the preclusion of any contributions of ELMs on the $\mathrm{W}$ erosion source, and $\mathrm{b}$ ) the uncertainties in the representation of the far-SOL particle- and energy-fluxes arriving at the first wall (a free outward directed decay parameter).

The global material transport code WallDYN has also been employed to estimate the level of fuel-retention for both JET-C and JET-ILW [50]. The calculations qualitatively reproduce the Be deposition patterns from the JET-ILW campaign: strong deposition on top of the HFS divertor apron and little or no deposition on the rest of the $\mathrm{W}$ divertor. The level of the estimated Be deposition is within a factor of 3-10 of the experimentally measured values [51]. WallDYN results in a factor 10 lower fuel retention rates in JET-ILW compared to JET$\mathrm{C}$ which is in accordance with the experimental data from gas balance measurements [4].

Whereas for the contribution of ELMs on the material source a more integrated modelling approach is necessary and in principle possible (c.f. next section) the extrapolation of the plasma fluxes is technically difficult. Attempts to match main-chamber Langmuir probe data with EDGE2D-EIRENE simulations resulted in strong discrepancies in terms of the measured perpendicular ion-flux densities [52]. Apart from the uncertainties in the level of the anomalous transport this discrepancy can be related to the plasma not being in contact with the first wall in the code. A recent development of a new code SOLEDGE2D-EIRENE [53] has the potential to circumvent this problem. Unlike SOLPS or EDGE2D-EIRENE, this new code does have an extension of the plasma grid towards all bounding surfaces in the $2 \mathrm{D}$ 
poloidal plane (the vessel boundary). A critical issue here is to provide the code with proper boundary conditions for the plasma everywhere in contact with the vessel components. SOLEDGE2D-EIRENE employs the penalization technique, which also includes an improved model of the Bohm-Chodura sheath for shallow field angles [54]. Ideally, codes like SOLEDGE2D could be also coupled with turbulence codes liked TOKAM3X [55] or BOUT $++[56]$ to leverage the need in anomalous transport models; however, such an attempt has not been made within the framework of the edge modelling activities at JET.

But even without a fully detailed representation of the background plasma available, a plausible reduction of complexity can also help with respect to physics understanding as it was successfully shown for example a comparative study of material transport and layer formation in JET-C (JET with a full carbon wall) and JET-ILW [57] using ERO. In this work the plasma background profiles in the divertor had been parametrised, guided by pre-existing modelling using EDGE2D-EIRENE and IR and LP measurements in the divertor. This allowed for a successful sensitivity study to address the significance of self-sputtering or prompt re-deposition effects on the formation of $\mathrm{C}$ or Be deposited layers, assuming a fixed value of $\mathrm{C}$ or Be influx, without actually running SOLPS or EDGE2D-EIRENE for all cases investigated.

In order to assess the risk of starting ITER operations with a full $\mathrm{W}$ divertor, a dedicated melt exposure experiment had been performed in the JET-ILW configuration [58]. JET is the only tokamak being able to produce ELMs large enough ( $>300 \mathrm{~kJ}$ per ELM, i.e. comparable to mitigated ELMs size expected in ITER) to facilitate melting of tungsten. In the H-mode experiment and by moving the outer strike point onto a dedicated leading edge of the horizontal target plate the base temperature was raised within $\sim 1 \mathrm{~s}$ to allow transient ELMdriven melting during the subsequent $0.5 \mathrm{~s}$. Almost $1 \mathrm{~mm}\left(\sim 6 \mathrm{~mm}^{3}\right)$ of $\mathrm{W}$ was moved by $\sim 150$ ELMs within 5 subsequent discharges. A key result was that significant material losses in terms of ejections into the plasma were not observed.

For a numerical assessment of the driving forces of the $\mathrm{W}$ melt damage process the $3 \mathrm{D}$ thermal model MEMOS for melt layer dynamics was utilized [59]. By taking the measured perpendicular heat flux arriving at the plate the code calculated the $3 \mathrm{D}$ motion of melted material along the surface in the "shallow water" approximation of the Navier-Stokes equations, taking into account surface tension effects, molten material viscosity and radiative losses from the surface. It was found $[60,58]$ that the main accelerators of the melt motion are related to plasma pressure gradients along the plate, as well as gradients in surface tension and the $\mathrm{JxB}$ force of currents (externally applied and by thermo-emission of electrons) crossing 
the melt layer within a strong magnetic field. Despite the rigorous set up of the model an artificial plasma shielding factor $\mathrm{f}_{\mathrm{s}}=-0.3-0.4$ was necessary (being applied to the heat flux reaching the exposed edge of the $\mathrm{W}$-lamella) to match the experimental data (in both, $\mathrm{L}$ - and $\mathrm{H}$-mode discharges). A clear physics understanding of the mitigation factor $\mathrm{f}_{\mathrm{s}}$ is lacking. Due to strong electric fields arising at the leading edge gyro-orbit effects for particles reaching behind the protruding edge can be significant and are capble to redistribute the power load away from the surface into gaps (the Larmor smoothing effect [61]). However, these effects cannot explain fully the need of a heat flux mitigation factor in the MEMOS melt simulations.

\section{Integrated modelling approaches}

To address the impact of any changes in the core plasma performance on the particle and power exhaust and, vice-versa, the effect of any changes of the edge and divertor conditions on upstream conditions and thus the pedestal performance, integrated code suites like the JINTRAC code [62] have been applied. In JINTRAC the 2D EDGE2D-EIRENE code is coupled to the 1.5D JETTO-SANCO core code $[63,64]$ and radial heat and particle fluxes (plasma and neutrals) are exchanged at a common boundary (i.e. the separatrix) and redistributed in poloidal direction [65].

In advance of the actual installation of the ILW into JET predictive simulations for seeded type-I ELMy H-mode JET-ILW discharges had been executed using JINTRAC [66] and in order to assess quantitatively the expected W-concentration in the plasma core the JINTRAC results for power and flux transients at the PFCs were handed over to the DIVIMP code. As a result it was shown predictively that $\mathrm{Ne}$ seeding can indeed replace $\mathrm{C}$ as main radiator and that subsequently the $\mathrm{W}$-concentration can be kept low enough $\left(\mathrm{c}_{\mathrm{W}}<10^{-5}\right)$ in order to avoid a radiative energy quench of the main plasma.

The modelling of the full JET type-I ELM cycle in [66] has also led to the conclusion that the fluid approximation for the parallel transport in SOL-part model of JINTRAC (i.e. EDGE2D-EIRENE) is in fact sufficient to reproduce the energy balance in terms of the ELMwetted area and power in-out asymmetry. The peak power load obtained during the ELM however was overestimated by almost an order of magnitude compared to the experiment while the power-decay time at the target was underestimated accordingly making the power balance consistent / balancing the spatially and temporarily integrated ELM power loads. Indeed, earlier 1D PIC simulations have shown [67] that during the ELM the fluid approximation for the parallel SOL transport should be not applicable because kinetic effects 
play a significant role. From the PIC simulations derived time-dependent parametrisations of sheath-heat transmission coefficients and kinetic flux-limiting coefficients for an ELM-cycle had been implemented into EDGE2D-EIRENE and it was shown [68] that with the kinetic corrections included the EDGE2D-EIRENE simulated heat-flux rise time could be extended and thus led to lower peak power loads too. However it was also shown that the experimentally observed, long ELM durations of few ms as seen in the JET-ILW (e.g. [69]) cannot be reproduced by the code. It is suggested that this is potentially related to delays in the local recycling at the $\mathrm{W}$ target plates during the ELM-crash [38]. A detailed analysis of the effect of a change in the recycling on W PFCs is ongoing and is an active field of research. New codes addressing the effect of particle trapping and transient outgassing effects in PFCs are in development [70].

\section{Code developments and benchmarks}

Only recently the new code package SOLPS-ITER was developed [71] with the goal to combine the most recent version of MPI-parallelized EIRENE [72] with the B2.5 multi-fluid plasma computational fluid dynamics (CFD) code. The latter has been originally part of the SOLPS5.2 package [73] that includes a state-of-the-art drifts and edge current model. The new SOLPS-ITER code has been benchmarked against existing SOLPS4 simulations for the ITER divertor design and the results were presented in [71]. SOLPS-ITER is now furtherly improved by adding new features and it should be made available to a broader public to replace potentially any previous SOLPS version. One new SOLPS-ITER feature being currently developed is the extension of the plasma grid up the first wall [74]. Another added feature will be a dynamic particle inventory model [75] to address retention effects. Other couplings with material codes are in preparation, too. The presence of the new code package SOLPS-ITER certainly does not remove the need of a validation of the new code against the experiment. However a streamlined single version of the SOLPS code package is helpful in the view of benchmarks with other codes like EDGE2D-EIRENE or SOLEDGE2D-EIRENE.

The potential applications of integrated codes like JINTRAC or new developments like the ETS code (European Transport Solver [76]) are manifold. However, the use of such codes is complex because the posed problems are highly non-linear. Reduced models like the COREDIV code [77], which consists of a 1D core plasma code taking confinement times as input parameter and utilising a slab model for the SOL/divertor plasma can be useful for rapid 
estimates in system analysis codes for DEMO (e.g. [78, 79]). Some progress is seen in the validation of COREDIV for existing experiments (e.g. [80, 81, 82]); however a cross-code (JINTRAC, ETS) / cross-machine benchmark is still ongoing.

\section{Conclusion}

In view of the stride of the past JET edge modelling activities over the years [83, 84, 85], and even after the progress made more recently on the analysis of the power exhaust and plasmawall interaction issues for metallic devices, shown here for the JET-ILW, it is yet unclear whether a parametric scaling can (ever) be derived for the power exhaust problem. It is therefore unavoidable to further rely on validation, exploitation and improvement of existing edge codes against detailed measurements for predictions toward ITER or DEMO. It must be noted however that the predictability using 2D edge codes towards ITER oder DEMO is limited to the divertor region only, as ITER for example will be operated at low pedestal collisionality and the fluid treatment of the plasma might not be applicable anymore. Furthermore large uncertainties in the assumptions for pedestal transport exist. However, with "given" upstream input parameters assumptions a reasonable level of confidence in the divertor performance can be found for ITER ([31] and references therein). As a further remark we point out that ITER, as it will be using RMPs to mitigate the impact of ELMs, does constitute a 3D problem for particle and power exhaust to be solved with 3D codes like EMC3-EIRENE [86]. Existing 3D codes do also have their limitations, as for example EMC3-EIRNE can nowadays only be applied for a reduced operational window (i.e. attached divertor conditions) and there is a strong demand to furtherly develop and prepare EMC3EIRENE for future use.

Nevertheless, significant progress has been made in validating 2D egde codes with experiments addressing detachment and power exhaust in the JET-ILW. Indeed we acknowledge an improved understanding of the physics processes relevant detached divertor conditions that include atomic and molecular physics, impurity radiation about the detachment zone, and SOL flows and particle drifts. Under the assumption that the physics is correctly described in the model, the same codes must be applicable to all similar devices (with metallic walls, JET, AUG, Alcator C-mod). The validation process can only be complete if executed at all relevant devices. Despite the progress made however, the predictability of current $2 \mathrm{D}$ edge plasma models is still limited by unknowns and uncertainties 
in: the anomalous transport assumptions, the impact of neutrals on the parallel ion momentum balance (relevant for pressure loss in case of divertor detachment), stability of ionization front location within the divertor, non-coronal effects in impurity radiation distribution patterns, the density limit, to mention only a few. These aforementioned issues are regarded as hot topics in the research community (c.f. [16] suggested reading).

Beyond simulating the SOL/divertor plasma we also made progress in the field of plasma-wall interaction, i.e. on the modelling of impurity production and transport from PFCs at the first wall into the divertor. A strong advance is apparent on the completion and validation of the relevant PWI databases using MD codes. The application of PWI and PMI codes like ERO or WallDYN is successful and have become mature and reliable. The MEMOS modelling for $\mathrm{W}$ melt experiments can qualitatively explain the main drivers of the melt layer motion. Codes like DTOKS or DUSTRACK are being employed to model dust transport and migration. However any assessment of PWI/PMI issues is strongly linked with the edge plasma and cannot be seen isolated (i.e. linking the plasma particle flux redistribution with ERO or WallDYN, the impinging heat fluxes with MEMOS or the dust particle source with DTOKS/DUSTRACK which for the moment has large uncertainties, etc). The quality of any PWI/PMI code result depends on the quality of the underlying $\mathrm{SOL} /$ divertor plasma simulation (and vice-versa as PWI can have a significant impact on the plasma itself).

It is obvious that integrated modelling becomes more fashionable but due to its complexity it is not yet regarded as a reliable work-horse tool for the extrapolation to ITER or DEMO. It is of utmost importance that codes like JINTRAC or ETS are applied and exploited, for example, in preparation of a DT-campaign at JET after a dedicated validation process for full JET-ILW baseline scenarios.

\section{Acknowledgements}

This work has been carried out within the framework of the EUROfusion Consortium and has received funding from the Euratom research and training programme 2014-2018 under grant agreement No 633053. The views and opinions expressed herein do not necessarily reflect those of the European Commission. This work was also part-funded by the RCUK Energy Programme under grant EP/I501045. 


\section{References}

[1] S. Brezinsek et al., J. Nucl. Mater. 415 (2011) S936-S942

[2] G. F. Matthews et al., Phys. Scripta T145 (2011) 014001

[3] S. Brezinsek et al., J. Nucl. Mater. 463 (2015) 11-21

[4] S. Brezinsek et al., Nucl. Fusion 53 (2013) 083023

[5] V. Philipps et al., J. Nucl. Mater. 438 (2013) S1067-S1071

[6] T. Loarer et al., J. Nucl. Mater. 463 (2015) 1117-1121

[7] T. Eich et al., Phys. Rev. Lett. 107 (2011) 215001

[8] M. Makowski et al., Phys. Plasmas 19 (2012) 056122

[9] T. Eich et al., Nucl. Fusion 53 (2013) 093031

[10] A. Loarte et al., Nucl. Fusion 47 (2007) S203-S263

[11] A. Scarabosio et al., J. Nucl. Mater. 463 (2015) 49-54

[12] M. E. Fenstermacher et al., Plasma Phys. Contr. Fusion 41 (1999) A345-A355

[13] V. Kotov et al., Plasma Phys. Control. Fusion 50 (2008) 105012

[14] S. Wiesen et al., J. Nucl. Mater. 415 (2011) S535-S539

[15] C. Guillemaut et al., Nucl. Fusion 54 (2014) 093012

[16] M. Wischmeier et al., J. Nucl. Mater. 463 (2015) 22-29

[17] R. Schneider et al., Contrib. Plasma Phys. 46 (2006) 3-191

[18] R. Simonini et al., Contrib. Plasma Phys. 34 (1994) 368

[19] D. Reiter et al., J. Nucl. Mater. 196-198 (1992) 80

[20] S. Wiesen et al, ITC project report (2006), http://www.eirene.de/e2deir_report_30jun06.pdf

[21] M. Groth et al., J. Nucl. Mater 463 (2015) 471-476

[22] L. Aho-Mantila et al., Nuc. Fusion 52 (2012) 103006

[23] L. Aho-Mantila et al., J. Nucl. Mater. 438 (2013) S321-S325

[24] L. Aho-Mantila et al., Proc. 25th IAEA-FEC, St. Petersburg, Russia (2014)

[25] M. Groth et al., Nuc. Fusion 53 (2013) 093016

[26] M. Groth et al., Proc. 25th IAEA-FEC, St. Petersburg, Russia (2014)

[27] A. Chankin et al., "Influence of the ExB Drift in High Recycling Divertors on Target Asymmetries", accepted Plasma Phys. Control. Fusion (2015)

[28] C. Giroud et al., Proc. 25th IAEA-FEC, St. Petersburg, Russia (2014)

[29] A. Jarvinen et al., Nuc. Fusion (2015) subm.

[30] M .Groth et al., J. Nuc. Mater. 463 (2015) 471-476 
[31] A. Kukushkin et al., Fusion Eng. Des. 86 (2011) 2865

[32] D. Moulton et al., Proc. 42nd EPS Conference on Plasma Physics (2015)

[33] C. Gleason-Gonzales et al., Fus. Eng. Des. 89 (2014) 1042-1047

[34] S. Varoutis et al., Proc. 25th IAEA-FEC, St. Petersburg, Russia (2014)

[35] G. De Temmermann et al., these proceedings (PFMC 2015)

[36] G. F. Matthews et al., priv. communications

[37] C. Guillemaut et al., these proceedings (PFMC 2015)

[38] S. Brezinsek et al., these proceedings (PFMC 2015)

[39] A. Kirschner et al., Nucl. Fus. 40 (5) (2000) 989

[40] P. C. Stangeby, J. D. Elder, Nucl. Fusion 35 (1995)

[41] M. Bacharis et al., Phys. Plasmas 17 (2010) 042505

[42] M. Sertoli et al., J. Nuc. Mater. 463 (2015) 837-841

[43] K. Schmid et al., J. Nuc. Mater. 415 (2011) S284-S288

[44] D. Borodin et al., J. Nuc. Mater. 438 (2013) S267-S271

[45] C. Bjorkas et al., J. Nuc. Mater. 439 (2013) 174-179

[46] E. Safi et al., J. Nuc. Mater. 463 (2015) 805-809

[47] E. Safi et al., these proceedings (PFMC 2015)

[48] S. Brezinsek et al., Nucl. Fusion (2014) 103001

[49] K. Schmid et al., Nucl. Fusion 55 (2015) 053015

[50] K. Schmid et al., J. Nuc. Mater. 463 (2015) 66-72

[51] M. Meyer et al., these proceedings (PFMC 2015)

[52] C. Guillemaut, priv. Communication (2015)

[53] H. Bufferand et al., J. Nuc. Mater. 438 (2013) S445-S448

[54] P. C. Stangeby, Nucl. Fusion 52 (2012) 083012

[55] P. Tamain et al., Contr. Plas. Phys. 54 (2014) 555-559

[56] B. D. Dudson et al., J. Plas. Physics 81 (2015) 365810104

[57] A. Kirschner et al., J. Nuc. Mater. 463 (2015) 116-122

[58] J. W. Coenen et al., J. Nuc. Mater. 463 (2015) 78-84

[59] B. Bazylev et al., Physica Scripta T145 (2011) 014054

[60] B. Bazylev et al., pre-print submitted in Proc. 21st Int. Conference on Plasma Surface Interactions, Kanazawa, Japan, EFDA-JET-CP(14)01/06

[61] R. Dejarnac et al., Nucl. Fusion 54 (2014) 123011

[62] M. Romanelli, G. Corrigan, V. Parail, S. Wiesen et al., Plasma and Fusion Research 9 (2014) 
[63] G. Cenacchi, A. Taroni, (1988) JET-ITR(88)03

[64] L. Lauro-Taroni et al., Proc. 21st EPS I (1994) 102

[65] A. Taroni et al., Proc. 16th IAEA FEC, Montreal, Canada (1996)

[66] S. Wiesen et al., Plasma Phys. Control. Fusion 53 (2011) 124039

[67] D. Tskhakaya et al., Contrib. Plasma Phys. 48 (2008) 89-93

[68] D. Harting, S. Wiesen et al., J. Nuc. Mater. 463 (2015) 493-497

[69] E. de la Luna et al., Proc. 25th IAEA-FEC, St. Petersburg, Russia (2014)

[70] K. Schmid et al., these proceedings (PFMC 2015)

[71] S. Wiesen et al., J. Nuc. Mater. 463 (2015) 480-484

[72] D. Reiter et al, The EIRENE code, http://www.eirene.de

[73] V. Rozhansky et al., Nucl. Fusion 49 (2009) 025007

[74] W. Dekeyser et al., J. Nuc. Mater. 415 (2011) S584-S588

[75] X. Bonnin et al., J. Nuc. Mater. 390-391 (2009) 274-277

[76] D. P. Coster et al., Plasma Science IEEE Transactions, 38 (2010) 2085-2092

[77] R. Zagorski et al., Contrib. Plasma Phys. 48 (2008) 179

[78] M. Kovari et al., Fus. Eng. Des. 89 (2014) 3054-3069

[79] I. Ivanova-Stanik, J. Nuc. Mater. 463 (2015) 596-600

[80] G. Telesca et al., J. Nuc. Mater. 463 (2015) 577-581

[81] R. Zagorski et al., J. Nuv. Mater. 463 (2015) 649-653

[82] G. Telesca et al., J. Nuc. Mater. 438 (2013) S567-S571

[83] D. P. Coster et al., J. Nuc. Mater. 313-316 (2003) 868-872

[84] W. Fundamenski et al., Contrib. Plasma Phys. 48 (2008) 190-195

[85] M. Wischmeier et al., J. Nuc. Mater. 415 (2011) S523-S529

[86] O. Schmitz et al, "Three-dimensional modeling of plasma edge transport and divertor uxes during application of resonant magnetic perturbations at ITER", subm. Nucl. Fusion (2015) 